\title{
Istrés laboral como reto y como presión: Su relación con características de la personalidad
}

Occupational stress as challenge and pressure: Its relationship with personality traits.

\author{
Magdalena Escamilla Quintal \\ Jouinie Arias Aldana \\ Reinaldo Novelo Herrera \\ Universidad Autónoma de Yucatán
}

\begin{abstract}
Resumen
Generalmente, la psicología se ha centrado en lo patológico del estrés (Siegel \& Schrimshaw, 2000; Peiró, 2008). Sin embargo, desde la psicología positiva se enfatizan las emociones, actitudes y acciones orientadas al bienestar y a la obtención de lugares de trabajo positivos (Simmons \& Nelson, 2007). No obstante, ambas perspectivas no son excluyentes, ya que se ha puesto de manifiesto que las emociones positivas pueden coexistir con las negativas durante circunstancias estresantes y adversas. El objetivo del presente estudio fue analizar las relaciones existentes entre la valoración/percepción como presión o como reto de los potenciales estresores y características de personalidad: el optimismo, la resiliencia y el locus de control. Participaron 117 profesores de nivel medio superior y superior de Mérida, Yucatán, México. Los resultados muestran que el optimismo correlaciona positivamente con la dimensión responsabilidad personal percibida como reto y negativamente con las dimensiones carga de trabajo y balance casa/trabajo percibidas como presión. La resiliencia correlaciona positivamente con la dimensión relaciones en el trabajo, percibida como reto, y el locus de control correlaciona negativamente con las dimensiones relaciones en el trabajo y balance casa/trabajo percibidas como presión. Se discuten los resultados, aportaciones y limitaciones.
\end{abstract}

Palabras clave: Estrés como reto, estrés como presión, optimismo, resiliencia, locus de control.

Nota del Autor:

Magdalena Escamilla Quintal, Facultad de Psicología, Universidad Autónoma de Yucatán; Jouinie Arias Aldana, Facultad de Psicología, Universidad Autónoma de Yucatán; Reinaldo Novelo Herrera, Facultad de Psicología, Universidad Autónoma de Yucatán.

La correspondencia en relación con este artículo deberá dirigirse a Magdalena Escamilla Quintal, Facultad de Psicología, Universidad Autónoma de Yucatán, Calle 31A x 8, No. 300, Col. San Esteban, C.P. 97149, Mérida, Yucatán, México.

Correo electrónico: m.escamilla@uady.mx 


\section{Abstract}

Generally, psychology has focused on pathological stress (Siegel \& Schrimshaw, 2000; Peiro, 2008). However, positive psychology emphasizes emotions, attitudes and actions aimed at obtaining happiness and positive workplaces (Simmons \& Nelson, 2007). Nevertheless, both perspectives do not oppose each other since -as it has been shown- positive emotions may coexist with negative ones during stressing and adverse circumstances. The aim of this study was to analyze the relationship between appraisal/perception as pressure or challenge stressors and personality traits: optimism, resilience and locus of control. One hundred and seventeen high school and college teachers at Merida, Yucatan, Mexico participated in the study. The findings showed that optimism correlates positively with personal responsibility perceived as challenge, and negatively with workload and balance work/home; resilience correlated positively with relationships at job and locus of control correlated negatively with relationships at job and balance work/home. Findings, contributions and limitations are discussed.

Keywords: Stress as challenge, stress as pressure, optimism, resilience, locus of control.

Durante las últimas décadas, importantes transformaciones como la globalización de la sociedad y la economía, el desarrollo de nuevas tecnologías de información y comunicación, tienen un impacto importante sobre el trabajo y las organizaciones en general. En este sentido, una de las organizaciones e instituciones en donde existe una mayor complejidad de relaciones por la pluralidad de actores sociales es la educativa (docentes, administrativos, alumnos, padres de familia, etc.). Desde esta perspectiva la prevención de riesgos psicosociales resulta un reto para cualquier sociedad.

Resulta importante que los profesores sean precursores de pautas y modelos de comportamientos que promuevan el bienestar psicosocial; por lo que, es fundamental que experimenten una adecuada calidad de vida y salud en los diferentes ámbitos de desarrollo: personal, social y laboral.

El trabajo docente posee un alto nivel de complejidad, la que aumenta cada día a medida que la sociedad se va complejizando. Es así como las demandas de distintos actores sociales hacia los profesores se vuelven más sofisticadas (Selamé, 1998). Dadas las múltiples demandas a las que son sometidos los profesores en este contexto, es posible considerar a la docencia como una profesión estresante, donde se exige una interacción constante y absorbente, y que además enfrenta condiciones laborales insatisfactorias (Asún, Molina, \& Real, 2002). Cambios en el estatus profesional, en las posibilidades de realización personal y en el mantenimiento de la salud de los profesores son elementos clave para comprender la situación.

En la actualidad, la salud ya no es definida únicamente como ausencia de enfermedad sino como la construcción de un bienestar integral, donde lo físico, lo emocional y lo social resultan ser parte de un todo. Para esta nueva conceptualización de la salud la prevención resulta un eje central, lo remedial no es suficiente, identificar poblaciones vulnerables resulta importante. La preocupación por la prevención de riesgos psicosociales en el caso de los profesores y desde el interés por la educación de las generaciones jóvenes en un nuevo contexto económico, cultural y social, es urgente la intervención para que dichas metas sean alcanzadas.

De Frutos, González, Maillo, Peña y Riesgo (2007) opinan que un buen entorno laboral afecta a las personas y a los procesos de trabajo, mejorando en consecuencia los resultados, y agregan que en la actualidad son frecuentes las noticias que destacan el estrés y el malestar del profesorado. 


\section{Conceptualización y estudio del estrés}

En 1950 Hans Selye definió el término estrés, en su obra Stress, como una condición interna del organismo que se produce como respuesta ante agentes evocadores; este fenómeno ha sido estudiado desde distintos enfoques, en el ámbito personal y profesional, con diversas poblaciones de estudio y ocupaciones, así como en un nivel individual y colectivo. Desde una perspectiva transaccional, entre persona y ambiente, este fenómeno sólo se caracteriza adecuadamente si se tienen en cuenta la situación ambiental, así como la relación de la persona con respecto a esa situación a lo largo del tiempo (Peiró, 2000).

Asimismo, algunos autores utilizan el término para designar al proceso en el cual algunas características de la situación producen resultados negativos para el bienestar o salud de las personas (Mingote \& Pérez, 2003; Williams \& Cooper, 2004). De igual manera, Salanova (2003) considera al estrés laboral como un proceso en donde intervienen gran diversidad de estresores o demandas laborales y también recursos, tanto de la persona como del trabajo, cuya ausencia pueden convertirse en un estresor más, y su presencia puede amortiguar los efectos dañinos de los estresores. Éste último planteamiento, que concibe al estrés como un proceso, es el que en la actualidad se acepta como el más completo.

\section{Enfoque tradicional}

La investigación sobre el estrés ha estado dominada durante muchos años por un enfoque centrado en déficit, las disfunciones y las patologías; una perspectiva negativa de daño o pérdida (Vera, 2006). Dicha investigación ha focalizado su atención en los resultados negativos, lo que ha llevado a que el estrés se convirtiera en sinónimo de distrés, y que ambos conceptos se utilicen indistintamente. (Le Ferve, Matheny, \& Kolt, 2003).

\section{Enfoque de la Psicología Positiva}

La psicología positiva ha sido definida y desarrollada como el estudio de las emociones, los estados y las instituciones positivas. Tiene como objetivo promover un cambio en el foco de preocupación de la psicología, así como la promoción de las cualidades positivas (Seligman \& Csikszentmihalyi, 2000). Las emociones positivas se caracterizan por dar lugar a estados afectivos placenteros y beneficiosos para las personas; por otra parte, las organizaciones positivas hacen referencia a las características de los buenos trabajos y de las mejores organizaciones para trabajar, es decir, organizaciones que aprenden, innovadoras, imaginativas y adaptativas a los cambios futuros (Salanova, Martínez, \& Llorens, 2005).

En general, este enfoque tiene un impacto importante en la investigación sobre el estrés, recuperando los términos y los aspectos positivos que intervienen en dicho proceso y proponiendo un enfoque más integral en el estudio del mismo. De acuerdo con Peiró (2008), el enfoque de la psicología positiva aporta nuevas ideas para el diagnóstico del estrés laboral y la prevención de riesgos, en lugar de un análisis más amplio del estrés buscando sus efectos positivos y negativos. Por ejemplo, las diferentes creencias acerca de la felicidad pueden influir en la evaluación del estrés y sus interpretaciones como amenazas o reto.

\section{Características de personalidad}

Toda la investigación ha tomado al individuo como unidad focal de análisis del fenómeno. El estrés, para toda esta investigación, es una experiencia individual, aunque puede estar influida por fenómenos sociales. El estrés resulta ser una experiencia individual, por lo tanto. es importante identificar las diferencias individuales que permiten distintas respuestas y niveles de tolerancia ante las experiencias de estrés (Peiró, 2001).

Entre las características de personalidad que han sido más estudiadas, en la relación con el estrés, se encuentran el optimismo (Maruta, Colligan, Malinchoc, \& Offord, 2000), la resiliencia (Ryff \& Singer, 2003; Tugade \& Fredrickson, 2004; Vera, Carbelo, \& Vecina, 2006) y el locus de control (Glazer, Stetz, \& Izso, 2004; Kirkcaldy, Shepard, \& Furnham, 2002). Debido a lo anterior, hemos seleccionado estas características de 
personalidad como variables de este estudio. A continuación se describirán cada una de ellas.

Las investigaciones dentro de la psicología positiva han determinado que los sujetos optimistas tienen mejor calidad de vida, menos ansiedad y estrés, además de enfermarse menos. (Ambrós, 2008). El optimismo que puede definirse en términos de expectativas positivas generalizadas de resultado, como la creencia de que el futuro depara más éxitos que fracasos (Scheier, Carver, \& Bridges, 1994).

Los sujetos optimistas manifiestan un estilo de afrontamiento del estrés definido en términos de una mayor utilización de estrategias de afrontamiento activo (resolución de problemas y reestructuración cognitiva); en contraposición los pesimistas, optan por las estrategias de afrontamiento pasivo especialmente la autocrítica (Martínez-Correa, Reyes, García-León, \& González-Jareño, 2006).

Por otra parte, las personas resilientes son aquellas que ante la adversidad se doblan pero no se rompen, son capaces de experimentar emociones positivas en situaciones estresantes, tienen una gran fortaleza para resistir las adversidades de la vida, encuentran el sentido incluso a las experiencias más difíciles, mantienen su equilibrio psicológico y funcionan de un modo normal ante un suceso traumático (Fredrickson, 2001; Tugade \& Fredrickson, 2004). La resiliencia surge como concepto de investigación en la psicología positiva, intentando determinar porque algunas personas consiguen aprender de sus experiencias e incluso encontrar beneficios en ellas (Vera et al., 2006). La resiliencia está asociada con una tendencia a percibir los eventos potencialmente estresantes de una forma menos amenazadora (Tugade \& Fredrickson, 2004).

El término locus de control se refiere al lugar donde, desde la óptica del propio individuo, se origina la fuerza motivadora que lo lleva a actuar de una manera y determina los acontecimientos de su vida (Durán-Ramos, 2001).La construcción psicológica del locus de control procede de la teoría del aprendizaje social Rotter (1966).
Se denomina "internas" a las personas que perciben que sus acciones, y las consecuencias de éstas, dependen de sus propios recursos y competencias; mientras que las "externas", en general, perciben sus acciones como resultado de factores externos, como por ejemplo, de otras personas, de la suerte, o un accidente (Kirkcaldy, Petersen, \& Hübner, 2002; Kirkcaldy et al., 2002; Muhonen \& Torkelson, 2004). Para la psicología orga-nizacional, la diferenciación entre locus interno y externo es importante para explicar cómo los empleados enfocan el trabajo, tanto en sus actitudes como en sus conductas (Judge \& Bono, 2001).

Por tanto, el objetivo del presente estudio fue analizar las relaciones entre la valoración/ percepción como presión o como reto de los potenciales estresores y características de personalidad: el optimismo, la resiliencia y el locus de control.

\section{Método}

\section{Participantes}

Participaron 117 profesores de nivel medio superior y superior de la ciudad de Mérida, Yucatán, México: de los cuales 48 son hombres y 69 son mujeres. Cuentan con una media de edad de 41.37 años. Con respecto al estado civil, el $47 \%$ de la muestra es soltera y el $53 \%$ es casada. En lo referente al nivel de estudios, el 23.9\% tiene estudios de licenciatura, el $64.1 \%$ tiene estudios de maestría, el 3.4\% posee el título de doctor.

\section{Instrumentos}

Presión y Reto. Se utilizó una escala basada en el Pressures Management Indicator (PMI), compuesta por 21 ítems. Los ítems describen situaciones que pueden ser tanto fuentes de presión como de reto, por lo que se les pidió a los participantes que las calificaran según el grado de presión y el grado de reto que cada una de ellas representa. La escala de respuesta es tipo Likert con seis puntos de anclaje para presión, desde 1 ("Con toda evidencia no es una fuente de presión") a 6 ("Con toda evidencia es una fuente de presión"); para reto, desde 1 ("Con 
toda evidencia no es una fuente de oportunidad/ reto") a 6 ("Con toda evidencia es una fuente de oportunidad/reto").

La carga de trabajo se operacionalizó con una escala formada por 5 ítems. Un ejemplo de ítem es "Tener que trabajar muchas horas al día".

La responsabilidad personal se utilizó con una escala formada por 4 ítems. Un ejemplo de ítem es "Tener que asumir riesgos".

Las relaciones en el trabajo se midieron con una escala formada por 7 ítems. Un ejemplo de ítem es "La falta de comunicación en el trabajo".

El equilibrio casa/trabajo se calificó con una escala formada por 5 ítems. Un ejemplo de ítem es "La vida familiar con una pareja que también intenta desarrollarse profesionalmente".

Optimismo. Se utilizó la escala Life Orientation Test (LOT)- Reduced. Se trata de una versión reducida de 6 ítems. A los participantes se les indicó que dijeran en qué medida estaban de acuerdo con 8 frases acerca del trabajo y el empleo. Algunos ejemplos de ítems de esta escala son: "En tiempos de incertidumbre, generalmente pienso que me va a ocurrir lo mejor", "Siempre soy optimista en cuanto al futuro". La escala de respuesta es tipo Likert con cuatro puntos de anclaje, desde 1 ("Totalmente en desacuerdo") a 4 ("Totalmente de acuerdo"). Los ítems 2, 4 y 5 fueron invertidos de forma que una puntuación alta indica mayor optimismo.

Resiliencia. Esta variable se midió con una versión reducida de 10 items de la Ego-Resiliency Scale (ER-89) (Block \& Kremen, 1996). La escala de respuesta es tipo Likert con 4 puntos de anclaje, desde 1 ("Totalmente en desacuerdo") a 4 ("Totalmente de acuerdo"); de esta forma, una mayor puntuación indica un mayor grado de resiliencia.

Locus de Control. Se utilizó una escala unidimensional del locus de control adaptada a situaciones laborales. Se trata de una versión reducida de 8 ítems de la Work Locus de Control.
Se midió con la Work Locus of Control Scale (Spector, 2002). A los participantes se les indicó que dijeran en qué medida estaban de acuerdo con 8 frases acerca del trabajo y el empleo. Algunos ejemplos de ítems de esta escala son: "Un trabajo es lo que tú haces que sea", "Lograr el trabajo que quieres es en gran medida cuestión de suerte". La escala de respuesta es tipo Likert con seis puntos de anclaje, desde 1 ("Completamente en desacuerdo") a 6 ("Completamente de acuerdo"). Los ítems 2, 3, 4 y 6 fueron invertidos de forma que una puntuación baja indica un locus de control externo, mientras que una puntuación alta indica un locus de control interno.

\section{Procedimiento}

El diseño de investigación de este proyecto se realizó de acuerdo a cada una de los objetivos y metas. La selección de la muestra se realizó mediante un muestreo de extracción aleatoria simple de los centros participantes a partir de un listado con el total de los mismos. El tiempo promedio de la aplicación de este instrumento osciló en torno a los 30 minutos.

\section{Resultados}

La tabla 1 contiene los estadísticos descriptivos y la consistencia interna (alfa de Cronbach) de las variables del estudio.

Los coeficientes alfa de Cronbach $(\alpha)$ de cada subescala se presentan en la tabla 1, junto con las medias y las desviaciones estándar. Los índices a oscilan entre .22 y .82 (tabla 1).

Como se observa en la tabla 1 , con respecto a las variables de personalidad, podemos observar que los profesores son más resilientes que optimistas y que, en general, su locus de control es interno (rebasa la media teórica que indica que a mayor puntuación se tiene un locus de control interno). 
De acuerdo a los datos observados la responsabilidad personal es la dimensión de estrés laboral que más se percibe como reto; mientras que las Relaciones en el Trabajo es la dimensión de estrés laboral que se percibe como una presión (ver tabla 1).

La tabla 2 contiene las correlaciones entre las dimensiones del estrés laboral percibido como reto y las variables de personalidad: optimismo (1), resiliencia (2) y locus de control (3). Las correlaciones encontradas son significativas en los niveles de ${ }^{*} p \leq .05{ }^{* *} p \leq .01$.
Se puede observar que la variable optimismo correlaciona positivamente con la dimensión responsabilidad personal percibida como reto con un nivel de significancia al .01; lo que sugiere que cuanto mayor sean las expectativas positivas de los profesores, mayor reto representan las responsabilidades en el trabajo (ver tabla 2).

También es posible observar que la variable resiliencia correlaciona positivamente con la dimensión relaciones en el trabajo percibida como reto con un nivel de significancia al .05; lo que sugiere que establecer y mantener las relaciones en el trabajo a los profesores les significa un estrés laboral positivo (ver tabla 2).

Tabla 1

Medias, Desviaciones y la consistencia interna (alfa de Cronbach) de las variables de estudio

\begin{tabular}{lccc}
\hline & M & DT & $\alpha$ \\
\hline Optimismo & 3.17 & .518 & .22 \\
Resiliencia & 3.33 & .425 & .77 \\
Locus & 3.12 & .603 & .53 \\
\hline Retocarga & 3.67 & 1.03 & .54 \\
Retorelaciones & 3.82 & 1.05 & .69 \\
Retoresponsab & 4.45 & 1.19 & .74 \\
Retocasatrab & 3.61 & 1.33 & .71 \\
\hline Prescarga & 3.62 & 1.28 & .74 \\
Presrelaciones & 3.91 & 1.24 & 82 \\
Presresponsab & 3.59 & 1.40 & .80 \\
Prescasatrab & 3.19 & 1.32 & .72 \\
\hline
\end{tabular}

Tabla 2

Correlaciones entre el Estrés Laboral percibido como Reto y el Optimismo(1), la Resiliencia(2) y el Locus de Control(3) $^{(2)}$

\begin{tabular}{lccc}
\hline & $\mathbf{1}$ & $\mathbf{2}$ & $\mathbf{3}$ \\
\hline 4.RetoCarga & .017 & .179 & .043 \\
5.RetoRelaciones & .139 &. $\mathbf{1 9 8}$ & .043 \\
6.RetoResponsabilidad &. $\mathbf{2 9 9 ^ { * * }}$ & .165 & .025 \\
7.RetoCasa/Trab. & .111 & .015 & -.072 \\
\hline${ }^{*} \mathrm{p} \leq .05{ }^{* *} \mathrm{p} \leq .01$ & & &
\end{tabular}


La tabla 3 contiene las correlaciones entre las dimensiones del estrés laboral percibido como presión y las variables de personalidad: optimismo (1), resiliencia (2) y locus de control (3). Las correlaciones encontradas son significativas en los niveles de ${ }^{\star} p \leq .05{ }^{* *} p \leq .01$

Se puede observar que la variable optimismo correlaciona negativamente con las dimensiones carga de trabajoy balance casa/trabajo percibidas como presión al .05 de nivel de significancia; lo que demuestra que los profesores, cuando no esperan resultados positivos ante la carga de trabajo y equilibrar sus tiempos y roles entre este y la familia, les significa un estrés laboral negativo (ver tabla 3).

Cabe hacer hincapié que la dimensión responsabilidad personal es percibida como reto y correlaciona positivamente (con un nivel de significancia al .01) y como presión correlaciona negativamente (con un nivel de significancia al .05) con la variable optimismo; sin embargo, el puntaje es más alto cuando es percibida como reto (ver tabla 2).

Por otro lado, la variable locus de control correlaciona negativamente con las dimensiones relaciones en el trabajo (con un nivel de significancia al .01) y el equilibro entre el trabajo y el hogar percibidas como presión (con un nivel de significancia al .05) lo que sugiere que los profesores, con un menor locus de control (o externo), tienden a percibir negativamente las relaciones en el trabajo y el justo equilibrio entre los tiempos y roles entre la familia y el trabajo. La variable resiliencia no correlaciona significativamente con ninguna de las dimensiones de estrés laboral percibido como presión (ver tabla 3).

\section{Discusión}

El análisis de la literatura sobre el proceso del estrés laboral demuestra estar dominada por un enfoque tradicional, centrado en lo patológico, de "enfermedad" o "daño". La mayoría de las investigaciones se han centrado en estudiarlo como un fenómeno negativo (distrés) que afecta a la salud y repercute en el bienestar de las personas. Sin embargo, actualmente surgen nuevas alternativas para el estudio del mismo, éstas tratan de ver el lado positivo del estrés, con la finalidad de entender la psicología y la salud mental (Vera, 2006).

Por lo anterior, el objetivo del presente estudio fue analizar las relaciones existentes entre la valoración/percepción, como presión o como reto de los potenciales estresores y características de personalidad: el optimismo, la resiliencia y el locus de control.

Se ha mencionado que las personas que poseen altos niveles de esperanza están relacionados con un mejor desempeño académico y deportivo, así como en los

Tabla 3

Correlaciones entre el Estrés Laboral percibido como Presión y el Optimismo ${ }^{(1)}$, la Resiliencia ${ }^{(2)}$ y el Locus de Contro/(3)

\begin{tabular}{lccc}
\hline & 1 & 2 & 3 \\
\hline 4.PresiónCarga & $-.204^{*}$ & -.092 & -.082 \\
5.PresiónRelaciones & -.133 & -.036 &.$- .271^{* *}$ \\
6.PresiónResponsabilidad & $-\mathbf{- 1 8 7 ^ { * }}$ & -.093 & -.151 \\
7.PresiónCasa/Trab. &.$- .226^{*}$ & -.013 & $-.194^{*}$ \\
\hline${ }^{*} p \leq .05{ }^{* *} p \leq .01$ & & &
\end{tabular}


resultados de procesos terapéuticos y la salud física (López, 2005). Lo anterior apoya los resultados del presente estudio; puesto que, los profesores con mayor optimismo tienden a percibir las diversas situaciones (dimensiones) de trabajo y particularmente la responsabilidad personal como un reto; los menos optimistas perciben como presión la carga de trabajo y el balance entre los tiempos y roles del trabajo y el hogar.

Asimismo, la resiliencia está asociada con una tendencia a percibir los eventos potencialmente estresantes de una forma menos amenazadora (Tugade \& Fredrickson, 2004) es probablemente lo argumentado por estos autores. En este estudio los profesores con mayor resiliencia perciben como un reto las relaciones en el trabajo en donde son determinantes los procesos de comunicación, el sentirse valorado y enfrentar la situación de discriminación o favoritismo.

Con respecto al locus de control, los profesores con un locus interno perciben como un reto las relaciones en el trabajo y el equilibrio entre los tiempos, roles entre la casa y el trabajo. Lo anterior, es congruente con lo encontrado por algunos autores; las personas con un locus de control interno tienen menos probabilidad de sufrir los efectos adversos del estrés. Esto contrasta con las personas con un locus de control externo que experimentan altos niveles de estrés, con secuelas negativas para la satisfacción en el trabajo, la salud física y mental (Kirkcaldy et al., 2002; Spector, 2002).

El presente estudio muestra ciertas limitaciones. El uso exclusivo de medidas de autoinforme que plantea el problema de la varianza común del método; es decir, la varianza atribuible al procedimiento de medida más que a las variables consideradas. Por otro lado, está el número de participantes, por lo que se sugiere que la exploración para futuras investigaciones se realice con muestras de mayor tamaño.

Finalmente, los hallazgos obtenidos en el presente trabajo suponen un avance en la clarificación de las interrelaciones entre las distintas valoraciones/percepciones del estrés laboral (positivas y negativas) y características de la personalidad. 


\section{Referencias}

Ambrós, V. (2008). El pesimismo se aprende y el optimismo también. Recuperado de http:// destres.wordpress.com/tag/el-optimismo-seaprende/

Asún, D., Molina, D., \& Real, C. (2002). Burnout en educación. Recuperado de http://www.educarchile.cl/Portal.Base/Web/ VerContenido.aspx?ID=74768

Block, J., \& Kremen, A. (1996). IQ and egoresiliency: conceptual and empirical connections and separateness. Journal of Personality and Social Psichology, 70(2), 349361.

De Frutos, J., González, P., Maillo, A., Peña, J., \& Riesco, M. (2007). Condiciones de trabajo y Satisfacción Laboral de los docentes en las escuelas católicas de Madrid. Educación y Futuro, 17, 9-42.

Duran-Ramos, T. (2001). El constructo locus de control en la toma de decisiones educativas. Paedagogium, 1(3), 8-11.

Fredrickson, B. L. (2001). The role of positive psychology: The broaden-and-build theory of positive emotions. American Psychologist, 56, 218-226.

Glazer, S., Stetz, T. A., \& Izso, L. (2004). Effects of personality on subjective job stress: A cultural analysis. Personality and Individual Differences, 37, 645-658.

Judge, T. A. \& Bono, J. E. (2001). Relationship of core self-evaluations traits-self-esteem, generalized self efficacy, locus of control, and emotional stability-with job satisfaction and job performance: A meta-analysis. Journal of Applied Psychology, 86, 80-92.

Kirkcaldy, B. D., Shepard, R. J., \& Furnham, A. F. (2002). The influence of type A behaviour and locus of control upon job satisfaction and occupational health. Personality and Individual Differences, 33, 1361-1371.
Kirkaldy, B. D., Petersen, L., \& Hübner, G. (2002). A comparison of occupational stress, physical and psychological well-being and coping amon managers from west and the former east Germany. European Psychologist, 7(1), 53-62.

Le Ferve, M., Matheny, J., \& Kolt, G. S. (2003). Eustress, distress and interpretation in occupational stress. Journal of Managerial Psychology. 18(7), 726-744.

López, D. (2005). Estrés, cómo entenderlo, entenderse y vencerlo. $4^{\circ}$ ed. Argentina: Lumen.

Martínez-Correa, A., Reyes, G. A., GarcíaLeón, A., \& González-Jareño, M. I. (2006). Optimismo/pesimismo disposicional y estrategias de afrontamiento y estrategias de afrontamiento. Psicothema, 18(1), 66-72.

Maruta, T., Colligan, R.C., Malinchoc, M., \& Offord, K.P. (2000). Optimists vs. pessimists: Survival rate among medical patients over a 30-year period. Mayo Clinic Proceedings, 75, 140-143.

Mingote, J. C., \& Pérez, S. (2003). Estrés en la enfermería. El cuidado del cuidador. Madrid: Díaz de Santos.

Muhonen, T. \& Torkelson, E. (2004). Work locus of control and its relationship to health and job satisfaction from a gender perspective. Stress and Health: Journal of the International Society for de Investigation of Stress, 20, 21-8.

Peiró, J. M. (2000). Desencadenantes del estrés laboral. Madrid: Pirámide.

Peiró, J. M. (2001). El estrés laboral: una perspectiva laboral y colectiva. Prevención, Trabajo y Salud, 13, 18-38.

Peiró, J. M. (2008). Stress and coping at work. New research trends and their implications for practice. In K. Nâswall, J. Hellgren \& M. Sverke (Eds.), The individual in the changing working life (pp. 284-310). United Kingdom: Cambridge University Press. 
Rotter, J. B. (1996). Generalized expectancies for internal versus external control of reinforcement. Phychological Monographs. 80 (Whole No. 609, 1).

Ryff, C., \& Singer, B. H. (2003). Emotion, social relationships and health. New York: Oxford University Press.

Salanova, M. (2003). Trabajando con tecnologías y afrontando el tecnoestrés: el rol de las creencias de eficacia. Revista de Psicología del Trabajo y las Organizaciones, 19, 225-247.

Salanova, M., Martínez, I., \& Lorens, S. (2005). Psicología organizacional positiva. En F. J. Palací (coord.). Psicología de la organización (pp.349-376). Madrid: Pearson- Prentice Hall.

Selamé, T. (1998). Condiciones de trabajo y salud ocupacional de la región Metropolitana. Mujer y Trabajo, 2.

Seligman M. E. P. \& Csikszentmihalyi, M (2000). Positive Psychology. American Psychologist, 55, 1-14.

Selye, H. (1950). Stress. Montreal: Acta In.

Scheier, M. F., Carver, C. S., \& Bridges, M. W. (1994). Distinguishing optimism from neuroticism (and trait anxiety, self-mastery and self-steem): a reevaluation of Life Orientation Test. Journal of Personality and Social Psychology, 67, 1.063-1.078.

Siegel, K. \& Schrimshaw, E. W. (2000). Perceiving benefits in adversity: Stress-related growth in women living with HIV/AIDS. Social Science \& Medicine, 51, 1543-1554.

Simmons, B. L., \& Nelson, D. L. (2007). Eustress at work: Extending tha holistic stress model. In B. L. Simmons \& D. L Nelson (Eds.), Positive organizational behavior (pp. 41-53). India: Sage.
Spector, P. E. (2002). Employee control and occupational stress. Current Directions in Psychological Science, 11(4), 133.

Tugade, M. M. \& Fredrickson, B. L. (2004). Resilient individuals use positive emotions to bounce back from negative emotional experiences. Journal of Personality and Social Psychology, 86(2), 320-333.

Vera, B. (2006). Psicología positiva: Una nueva forma de entender la psicología. Papeles del Psicólogo, 27(1), 3-8.

Vera, B., Carbelo, B., \& Vecina, M. (2006). La experiencia traumática desde la psicología positiva: resiliencia y crecimiento postraumático. Papeles del psicólogo, 27, 4049.

Williams, S. \& Cooper, C. L. (2004). Manejo del estrés en el trabajo. México: Manual Moderno. 